\title{
Comparison of a modified thiazole orange technique with a fully automated analyser for reticulocyte counting
}

\author{
D Bowen, N Bentley, T Hoy, I Cavill
}

\begin{abstract}
Two independent methods for quantitating reticulocyte counts were compared. One used a modified thiazole orange technique and a flow cytometer (Becton Dickinson FACS); the other was a fully automated whole blood analyser (Sysmex R1000). Both methods gave comparable results with a coefficient of variation of less than $5 \%$. Samples measured using the $R 1000$ showed a negligible decrease in the reticulocyte count over five days at room temperature, although there was evidence of continuing intracellular maturation: with thiazole orange there was an apparent increase. A practical reference range of $20-70 \times 10^{9} / 1$ was established from 89 normal subjects.

The close correlation between the two independent estimates indicates the validity of the quantitation of the reticulocyte count and shows that automation allows significant changes within and below the normal range to be detected with a degree of reliability which was not previously possible.
\end{abstract}

The reticulocyte count has long been recognised as an important indicator of erythropoietic activity, but the semiquantitative visual methods available have not permitted anything more sophisticated than the detection of a grossly increased count. Significant changes within and near the normal range could not be detected, even though they may have represented twofold changes in erythroid production. The availability of RNA binding dyes and flow cytometry has created a resurgence of interest in reticulocyte counting. ${ }^{1}$ Most of the limitations have been overcome by automated reticulocyte counting, which removes inter-observer error, reduces the coefficient of variation by counting many more cells, and is faster.

Improved automated techniques have led to the development of the Sysmex R-1000 (Sysmex-TOA Electronics Company, Kobe, Japan). The most easily used non-automated method currently available entails staining with thiazole orange and subsequent analysis of samples by flow cytometry. We describe a modification of the thiazole orange technique aimed at reducing methodological variation and a comparison of this modification with the Sysmex R-1000.
Methods

THIAZOLE ORANGE

A stock solution of thiazole orange (Molecular Probes Inc, Eugene, Oregon, USA) $(1 \mathrm{mg} / \mathrm{ml})$ in methanol was diluted 1 in 10000 in phosphate buffered saline with $0.002 \mathrm{M}$ EDTA and $0.02 \%$ sodium azide. ${ }^{2}$ This solution was prepared fresh each week and stored at room temperature in the dark until used. Whole blood $(5 \mu \mathrm{l})$ was added to each of two tubes containing $1 \mathrm{ml}$ of thiazole orange solution. One sample was incubated at $20-23^{\circ} \mathrm{C}$ for between 30 and 55 minutes and the second for between 70 and 100 minutes. Unstained samples were incubated for the same lengths of time in each case.

After incubation the samples were analysed on a FACS 440 (Becton Dickinson). Two hundred thousand red cells were counted when evaluating the effects of temperature, incubation time, and storage. The forward light scatter pattern of the control sample was used to set gates to include only red cells and to exclude platelets and nucleated cells. Reticulocytes were defined as those cells with more than $0.3 \%$ fluorescence compared with unstained samples. Studies of different incubation times had shown near linear staining over this time period (fig 1) and the reticulocyte count was therefore calculated at a standard 60 minutes by interpolation from the counts observed at the two incubation times. This overcame the necessity to analyse the stained sample at precisely 60 minutes, which may not be possible in a busy laboratory. The absolute reticulocyte count for this method was calculated from the estimated percentage, and the red cell count was measured on a Technicon $\mathrm{Hl}$ automated counter.

The reticulocyte RNA specific fluorescence frequency distribution was also analysed to produce an index of reticulocyte maturity.

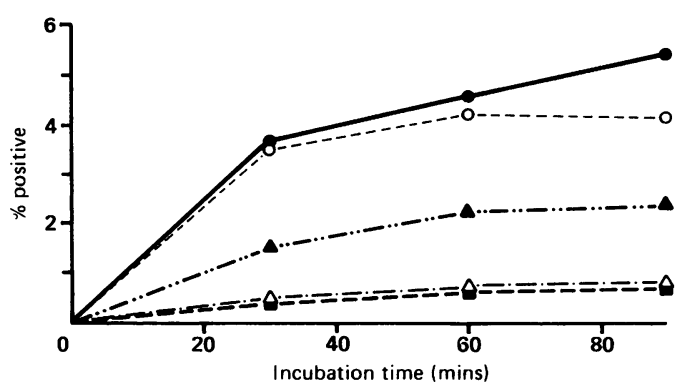

Figure 1 Effect of incubation time on thiazole orange staining for five blood samples. 
The mean RNA content of all reticulocytes was computed relative to a value of one for the unstained sample. The mean RNA content of the $10 \%$ most fluorescent cells was computed similarly. It was necessary to analyse one million cells to obtain sufficient numbers in this top $10 \%$ fraction.

SYSMEX R-1000

This fully automated flow cytometric reticulocyte counter uses an argon laser light source and a sheath flow system to determine cell size (forward scatter) and RNA content $\left(90^{\circ}\right.$ scatter) of peripheral blood cells. A $100 \mu \mathrm{l}$ sample of well mixed EDTA anticoagulated blood is sampled and stained with the nucleic acid fluorochrome, auramine-O. Gates are computed for each individual sample to exclude mature platelets and nucleated cells so that the fluorescent reticulocytes and the non-fluorescent mature erythrocytes are identified and counted. The fluorescent channels containing reticulocytes are divided into three to give the percentage of reticulocytes with high, moderate, or low fluorescence. At the same time the red cell count is also measured and the reticulocyte count is calculated directly as an absolute count $\left(10^{9} / 1\right)$.

EDTA anticoagulated blood was used for all analyses. Eighty nine blood samples from healthy normal donors were evaluated to define a normal range for the Sysmex R-1000. Twenty nine separate samples from a similar study group were stained and studied with thiazole orange to produce a normal range for this technique. Fifty nine samples from 43 patients, most of which were analysed as part of other studies, were also counted by both methods. Some patients were studied twice or more, but always on different days. All the patients, except six, were anaemic and included 27 with myelodysplasia, eight with chronic renal failure, two with haematinic deficiencies, and one each with autoimmune haemolysis, hypoplastic anaemia, and an unexplained dual red cell population: three of these samples were from normal subjects.

\section{Results}

REFERENCE RANGES

Thiazole orange

The percentage reticulocyte count in the 29 samples from normal subjects ranged from $0 \cdot 4$ to $2 \cdot 1$ with a mean (SD) of $1 \cdot 2(0.4) \%$. The absolute reticulocyte count was between 19 and 98 with a mean (SD) of $59(20) \times 10^{9} / 1$ and these contained $9 \cdot 7(2 \cdot 5)$ RNA units/reticulocytes while the top $10 \%$ of the most fluorescent cells had a mean (SD) RNA content of $36 \cdot 6$ $(17 \cdot 6)$ units.

\section{Sysmex R1000}

The reticulocyte count in the 89 normal subjects ranged between $0.5 \%$ and $1.7 \%$ with a mean (SD) of $1 \cdot 1(0 \cdot 3) \%$. The mean (SD) of $1 \cdot 1$ $(0.3) \%$ in the 37 men was the same as the mean (SD) of $1 \cdot 1(0.3) \%$ in the 48 women. The absolute reticulocyte count in these subjects ranged from 19 to $88 \times 10^{9} / 1$ with a mean (SD)

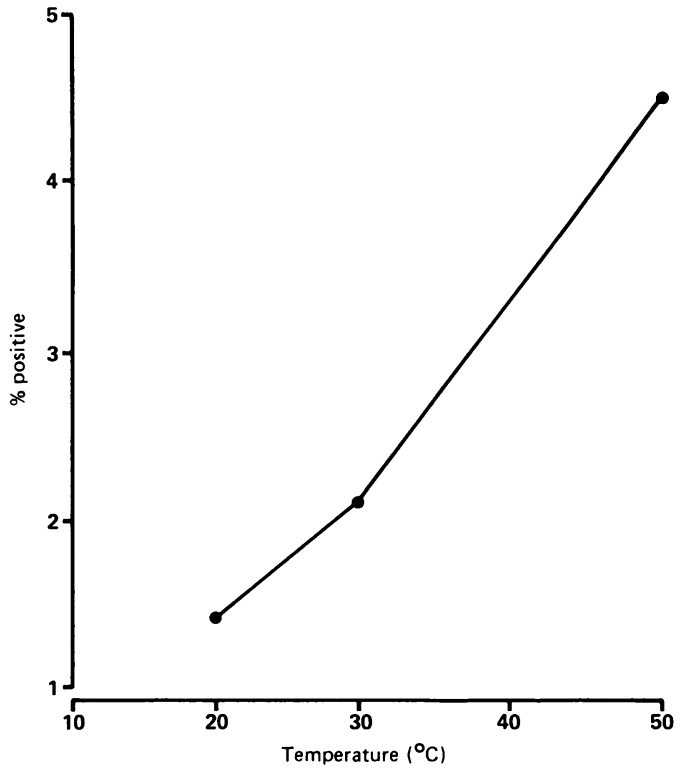

Figure 2 Mean percentage thiazole orange staining for aliquots of five samples incubated at different temperatures.

of $46(13) \times 10^{9} / 1$. Again, there was no significant difference between the mean (SD) for the men, 48 (12) $\times 10^{9} / 1$ and that for the women, $43(12) \times 10^{9} / 1$. The distribution of these data approximated to normal, with a median of $45 \times 10^{9} / 1$, and the practical reference range $e^{3}$ for this variable, which is used in this laboratory, is 20 to $70 \times 10^{9} / 1$. The proportion of reticulocytes containing high fluorescence ratio varied between $0 \cdot 1$ and $6.5 \%$ in these 89 subjects with a mean (SD) for the men of $2 \cdot 5(1 \cdot 1) \%$ and for the women of $2 \cdot 3$ $(1 \cdot 3) \%$.

\section{REPRODUCIBILITY}

The coefficient of variation (CV) for each method was assessed by the 10-fold replicate analysis of samples with normal or modestly increased reticulocyte counts. For the thiazole orange method this $\mathrm{CV}$ varied between 2.4 and $4.8 \%$ for 10 separate samples with a mean CV of $3.4 \%$. Similarly, for the Sysmex R1000 the $\mathrm{CV}$ for three separate samples was between 3.9 and $5.5 \%$ with a mean of $4.3 \%$.

TEMPERATURE AND THIAZOLE ORANGE STAINING The increase in fluorescence which occurs when thiazole orange penetrates cells and combines with intracellular nucleic acid is time

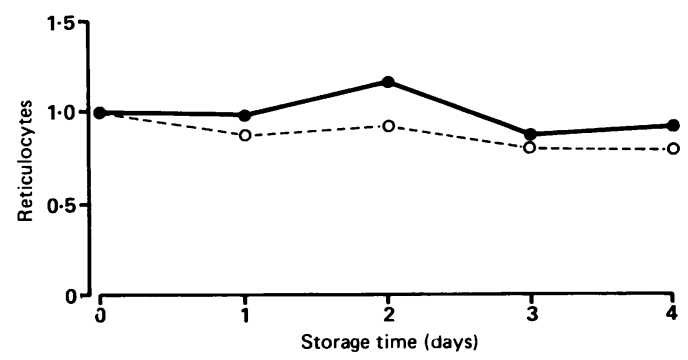

Figure 3 Effect of time on the normalised mean reticulocyte count measured with the Sysmex R1000 for samples stored at $4^{\circ} \mathrm{C}(\bullet, n=8)$ and room temperature $(0, n=15)$. 
Figure 4 Change in the mean percentage of cells with high fluorescence ratio (HFR) for 10 samples stored at room temperature.

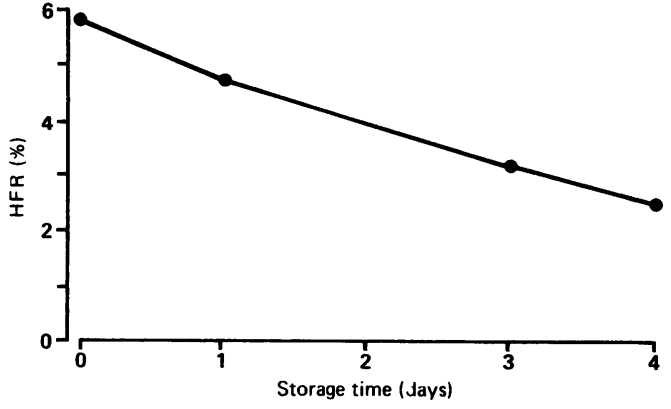

dependent. ${ }^{2}$ After an initial rapid increase in fluorescence there is a steady rise, which approaches, but does not reach, a plateau. The temperature of incubation of the cells and thiazole orange has a pronounced effect on the rate of reaction of the initial and prolonged phases (fig 2). In this study the reticulocyte count for patient samples was derived from incubation at 20,30 , and $50^{\circ} \mathrm{C}$.

\section{EFFECT OF STORAGE}

No significant change in reticulocyte percentage measured by the thiazole orange method was found for samples stored for up to 48 hours at $4^{\circ} \mathrm{C}$. Storage at room temperature caused the measured percentage reticulocyte count to increase up to 48 hours. This continued over the subsequent two days. For samples stored at $4 \mathrm{C}$ for up to four days the reticulocyte counts measured by the $\mathrm{R} 1000$ remained unchanged. At room temperature the percentage showed a small but significant $(p<0.05)$ fall after 24 hours (fig 3 ), but thereafter remained largely unchanged. At the same time the proportion of highly fluorescent cells decreased (fig 4) and there was a reciprocal increase in low fluorescent cells.

\section{COMPARISON OF THIAZOLE ORANGE AND SYSMEX R1000}

Reticulocyte counts obtained by the two methods were compared in 59 samples. The percentage reticulocyte count in these ranged between 0.2 and $6.8 \%$ and there was a good correlation between the two estimates $\left(r_{s}=0.805, p<0.01\right)$ (fig 5). The reticulocyte count was between 5 and $291 \times 10^{9} / 1$, and again there was a strong correlation between the two estimates $\left(r_{s}=0.797, p<0.01\right)$.

The two different approaches to assessing reticulocyte maturity were also compared in these patients. The analysis of the thiazole orange staining data which we have developed gives an estimate of the mean RNA content of the $10 \%$ most fluorescent cells. The Sysmex

Figure 5 Correlation of percentage reticulocyte count measured using both the thiazole orange method and the Sysmex R1000.

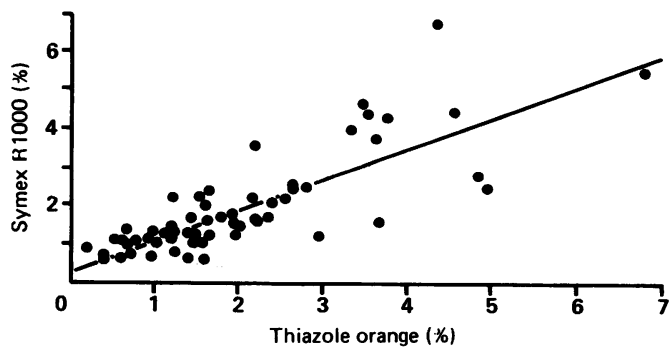

$\mathrm{R} 1000$ high fluorescence ratio is the percentage of the cells which fluoresce in the top one third of the range. There was a significant but weak correlation between these two variables $\left(r_{\mathrm{s}}=0.380, \mathrm{p}<0.05\right)$, but there was a stronger correlation $\left(r_{s}=0.430, p<0.01\right)$ between the high fluorescence ratio and the mean RNA index of the whole population of red cells with thiazole orange.

\section{Discussion}

This study has shown that using two independent staining techniques reticulocytes can be counted in whole blood samples with comparable results. A correlation between visual reticulocyte estimates and the Sysmex R1000 count has already been shown, ${ }^{45}$ and we have confirmed this locally ( $n=140, r=0.73$ ). This has established the qualitative link between the presence of reticulocytes and their detection by flow cytometry. The quantitative validity of a measure with a coefficient of variation of less than $5 \%$, however, is not established by comparison with a semiquantitative estimate with a coefficient of variation of greater than $25 \%$. This study shows the correlation between two independent quantitative estimates and lends support to the validity of the quantitation of reticulocyte counts by flow cytometry. Moreover, this has been shown in the normal and near normal range where the reliable detection of either decreased or increased reticulocyte production has thus far not been possible.

The choice of methodology seems to be determined by local circumstances. Where there is a general purpose flow cytometer it may be possible to take advantage of the sensitivity of thiazole orange. The modification of the method which we have described will remove much of the variability caused by lack of standardisation of temperature and time of incubation. The speed and simplicity of the $\mathrm{R} 1000$, however, allows estimates to be made directly in the laboratory as part of an integrated haematological investigation. In our laboratory the R1000 is linked on-line to the laboratory data processing system and is effectively available as an extension of the routine blood counting service.

The reference range which we have found using both thiazole orange and the R1000 is consistent with that found in similar studies elsewhere. ${ }^{46}$ The wide range in both percentage and absolute reticulocyte counts is a measure of the variability of erythropoietic activity and reticulocyte maturation time which may be found in normal subjects. This variation does not seem to be related to haemoglobin concentration. The separate measurement of the youngest reticulocyte proportion also offers a means of detecting and measuring rapid changes in the kinetics of erythropoietic output. The present appreciation of the value of the reticulocyte count is largely confined to its role as a detector of gross haemolysis. The ability to detect much smaller changes in the near normal range will permit a less simplistic appreciation of both the 
percentage and absolute counts. In particular, the ability to measure subnormal reticulocyte counts presents the possibility of anticipating the consequences of erythropoietic change.

A consistent finding in this study was that the reticulocyte count measured in a sample of stored blood did not disappear rapidly as might have been expected. Indeed, with our modified thiazole orange method the apparent count rose consistently over a matter of days at room temperature, and this has been observed in other studies. ${ }^{7}$ Whether this was due to changes in erythrocyte permeability leading to increased non-specific staining or some other factor is not clear. In contrast, the R1000 reticulocyte count, both percentage and absolute, remained stable at $4^{\circ} \mathrm{C}$ and showed only a small decline over five days at room temperature. This change was greater than could be accounted for by methodological variation and this has been found in another study. ${ }^{5}$ At the normal level this may account for a fall of $0.3 \%$. This suggests that for automated reticulocyte counts samples can be stored and, unlike visual counting methods, do not require immediate analysis. Our results suggest that the loss of measurable RNA does not continue at the same pace in vitro as it does in vivo where maturation is complete within one to two days. ${ }^{8}$

The maturation process is also generally assumed to involve loss of cell volume. This is based on the larger and polychromatic image that reticulocytes create compared with erythrocytes when both are smeared on a slide. The assessment of reticulocyte volume by both the thiazole orange method and the R1000, however, shows that this is not the case. While cells containing stainable RNA are usually clustered with the larger half of the unstained cells they are by no means the largest cells. At any given cell size they are always outnumbered by unstained erythrocytes. From these data it would be unwise to assume that the largest cells in any red cell distribution represent the reticulocytes. Even when there is increased reticulocyte production the same size distribution is still seen. Indeed, it may well be that the reticulocyte gives rise to an erythrocyte of similar size and that the large reticulocyte image on the blood film is another artefact of the preparative method.

The indices of reticulocyte maturity which we have developed for the analysis of the thiazole orange fluorescence distribution and the in-built analysis of the R1000 both show that there can be significant variation in the age structure of the reticulocyte population. In normal subjects the high fluorescence ratio is held within a narrow range, but when there is a stimulus to erythropoiesis, such as results from therapeutic $\mathrm{rHu}$ Epo, then there is a substantial increase in the mean RNA content of the reticulocytes. When the high fluorescence ratio is expressed as the number of reticulocytes then the increased production of the marrow is even more evident.

The reticulocyte count has long been recognised as a haematological variable, but the qualitative estimates have been unreliable. This study shows that this variable can now be reliably measured by flow cytometry and automation means that this can be routine. As yet the understanding and interpretation of these new variables are in the early stages, but they will probably yield information which can only be obtained by detailed ferrokinetic studies at present.

This study was supported by a grant from the Leukaemia Research Fund. The Sysmex R1000 was provided by LEP Scientific to whom we are particularly grateful.

1 Tanke HJ, Rothbarth PH, Vossen JMJJ, Koper GJM, Ploem JS. Flow cytometry of reticulocyte applied to clinical haematology. Blood 1983;61:109-17

2 Lee LG, Chen C-H, Chiu LA. Thiazole Orange: a new dye for reticulocyte analysis. Cytometry 1986;7:508-17.

3 Verwilgen RL. The use of reference values. In: Lewis SM Verwilgen RL, eds. Quality assurance in haematology. London: Baillière Tindall, 1988:33-41.

4 Kojima K, Niri M, Setoguchi K, Tsuda I, Tatsumi N. An automated optoelectronic reticulocyte counter. Am J Clin Pathol 1989;92:57-61.

5 Tichelli A, Gratwohl A, Driessen A, et al. Evaluation of the Sysmex R-1000. An automated reticulocyte analyzer. Am Sysmex R-1000. An automated

6 Carter JM, McSweeney PA, Wakeme PJ. Counting reticulocytes by flow cytometry: use of thiazole orange. reticulocytes by flow cytometry: us
Clin Lab Haematol 1989;11:267-71.

7 Ferguson DJ, Lee S-F, Gordon PA. Evaluation of reticulocyte counts by flow cytometry in a routine aboratory. Am J Haematol 1990;33:13-17.

8 Hillman RS. Characteristics of marrow production and reticulocyte maturation in normal man in response to anaemia. J Clin Invest 1969;48:443-53. 